Palabra Clave (La Plata), octubre 2017, vol. 7, n 1, e036. ISSN 1853-9912

Universidad Nacional de La Plata.

Facultad de Humanidades y Ciencias de la Educación.

Departamento de Bibliotecología

\title{
Usamos la información para optimizar actividades. El caso de un proyecto de extensión en una biblioteca popular
}

\author{
We use the information to optimize activities. A case of \\ an extension project in a popular library
}

\section{Elida Edith Elizondo *}

* Escuela de Bibliotecología, Facultad de Filosofía y Humanidades de la Universidad Nacional de Córdoba, Argentina | elidaeee@gmail.com

\section{PALABRAS CLAVE}

Bibliotecas Populares

Extensión universitaria

Comunicación

Gestión de la información

\section{KEYWORDS}

Popular libraries

University extension

Comunication

Information management

\section{RESUMEN}

Este artículo recoge resultados de un proyecto de extensión aplicado en la Biblioteca Popular Atahualpa de la ciudad de Córdoba, donde participaron estudiantes de la Escuela de Bibliotecología, Facultad de Filosofía y Humanidades (FFyH) de la Universidad Nacional de Córdoba (UNC). Involucradas las cátedras de "Administración de Recursos y Servicios de información I y II" y "Procesos Técnicos I" de la carrera, se consideró oportuno enfocarse en el uso de la información dentro del flujo comunicacional para contribuir en la necesidad de la biblioteca de ordenar el material bibliográfico donado afianzando conocimientos en los participantes. El objetivo se centró en valorizar la donación por medio de un análisis crítico del material, y durante el proceso se utilizaron algunas herramientas necesarias para informar, haciendo uso social de los conocimientos vertidos en la universidad. La intervención con un grupo de alumnos interesados en la práctica extensionista, fue la modalidad utilizada para asistir técnicamente a la biblioteca, además de la actividad- in situ de análisis crítico de la colección. Como resultado se percibió la importancia de utilizar adecuadamente la información para comunicar y responder al compromiso asumido, además del aprendizaje brindado a estudiantes y personal de la biblioteca.

\section{ABSTRACT}

This article collect results of an extension project applied in the Popular Library Atahualpa of the city of Córdoba, where participated students of Bibliotecología School, Faculty of Philosophy and Humanities (FFyH) of the National University of Córdoba (UNC). Involved the subjects of Administration of Resources and Services of Information I and II and Technical Processes I of the career, it was considered to be opportune to focus on the use of the information within the communication flow to contribute to the need of the library to order the donated bibliographic material secured knowledge in the participants. The objective was to value the donation through a critical analysis of the material, and during the process used some tools needed to inform, making social use of knowledge poured into the university. The intervention with a group of students interested in extensionist practice was the modality used to technically assist the library, in addition to the "on-site" activity of critical analysis of the collection. As a result, it was perceived the importance of using information properly to communicate and respond to the commitment assumed. 


\section{Introducción}

El desarrollo vertiginoso actual de la tecnología e información instala a las bibliotecas en un lugar de evaluación permanente en relación a las respuestas que se otorgan a las personas que recurren a ella. De esta manera, y para estar a la altura de las circunstancias, es necesario desarrollar múltiples acciones tendientes no sólo a organizar de manera adecuada una biblioteca sino para generar novedosos servicios o productos.

La cátedra "Administración de Recursos y Servicios de Información II" participa activamente en prácticas de extensión como rutina para volcar a la sociedad los conocimientos adquiridos en la Universidad Nacional de Córdoba (UNC), y en esta oportunidad ha dado respuesta a la solicitud de colaboración de la Biblioteca Popular Atahualpa.

Esta Biblioteca ubicada en el Barrio Marqués de Sobremonte de la ciudad de Córdoba, tomó la decisión de acudir a la Escuela de Bibliotecología informando acerca de la realidad en que se encontraba respecto a las donaciones mensuales que recibían de libros y revistas.

Estas donaciones no han tenido control ni límites, y por ende los espacios físicos de la biblioteca en cuestión se habían agotado. La intención de recuperarlos obligó a establecer una política en cuanto a las donaciones y a realizar un análisis crítico de las colecciones donadas.

La cátedra involucrada cursó invitación para participar a la cátedra "Administración de Recursos y Servicios de Información I" y "Procesos Técnicos I", de la carrera de Licenciatura en Bibliotecología y Documentación, que desarrollaron la actividad durante los meses de septiembre y octubre del año 2016.

De acuerdo a la complejidad, los responsables de la propuesta centraron su atención en diseñar actividades pertinentes al trabajo mismo, además de profundizar en contenidos para un mayor aprendizaje de los participantes seleccionando herramientas facilitadoras de información dentro de un marco de trabajo participativo.

\section{Metodología}

\section{Comenzar una práctica extensionista}

La decisión de intervenir en la biblioteca condujo al grupo conformado a evaluar y consensuar sobre las modalidades de trabajo que comprendieran todas las ideas propuestas.

La forma fue participativa, incluyendo a todos los integrantes en la realización de diagnóstico de la biblioteca, la tarea de definir el marco de trabajo, recolectar información y analizarla, establecer prioridades y redactar los objetivos que permitieran desarrollar la actividad. Los objetivos fueron los siguientes: 
Objetivo general:

- Valorizar colecciones donadas por medio de un análisis crítico de las mismas en la Biblioteca Popular Atahualpa, fomentando el compromiso social del profesional en bibliotecología.

Objetivos específicos:

- Establecer criterios de selección y guías de expurgo de la Biblioteca Popular

Atahualpa.

- Realizar una labor de selección de libros.

- Determinar el destino de los libros.

El comportamiento personal y las actitudes fueron factores primordiales para la integración y participación activa, lo mismo que el material bibliográfico seleccionado como lectura para el desarrollo de la propuesta.

Ante la decisión de aplicar un análisis crítico a las colecciones donadas, se organizó el trabajo en cuatro etapas bien diferenciadas secuencialmente y en forma general como plan de trabajo. La firme intención fue concretar dichas etapas paso a paso, y cumplir con el compromiso asumido con la institución y la Secretaría de Extensión de la FFyH-UNC. En la Tabla 1 se sintetiza lo planeado.

Tabla 1. Plan de Trabajo

\begin{tabular}{|l|l|}
\hline \multicolumn{1}{|c|}{ Actividad } & \multicolumn{1}{c|}{ Descripción } \\
\hline $\begin{array}{l}\text { Políticas de Expurgo } \\
\text { - Lectura de }\end{array}$ Bibliografía & $\begin{array}{l}\text { Dentro de los espacios institucionales es necesario } \\
\text { profundizar sobre conceptos y metodologías con la } \\
\text { finalidad de fortalecer saberes para su uso social. } \\
\text { El aporte realizado por los estudiantes en esta } \\
\text { práctica contribuye al desarrollo de sus habilidades y } \\
\text { alcanzar una formación integral. }\end{array}$ \\
\hline $\begin{array}{l}\text { Redacción de } \\
\text { políticas de Expurgo } \\
\text { para la Biblioteca } \\
\text { Popular Atahualpa }\end{array}$ & $\begin{array}{l}\text { Involucrarse en las decisiones institucionales y en este } \\
\text { caso en la redacción de políticas es un factor } \\
\text { determinante para que el personal sienta como propia } \\
\text { la institución y asuma un compromiso sólido con la } \\
\text { misma }\end{array}$ \\
\hline $\begin{array}{l}\text { Actividad de } \\
\text { Expurgo - }\end{array}$ & $\begin{array}{l}\text { La participación de los estudiantes y personal de la } \\
\text { biblioteca en esta actividad, genera un } \\
\text { involucramiento que le permite, en primera instancia } \\
\text { construir conocimientos desde la realidad y en } \\
\text { segundo lugar, influir en los procesos de las } \\
\text { instituciones con las cuales se vinculan para poder } \\
\text { transformar. }\end{array}$ \\
\hline $\begin{array}{l}\text { Evaluación de } \\
\text { Expurgo }\end{array}$ & $\begin{array}{l}\text { La actividad extensionista es un proceso de } \\
\text { intervención de carácter educativo por lo tanto, en } \\
\text { aquellos involucrados se produce un aprendizaje como } \\
\text { resultado de la experiencia. Esta asistencia técnica }\end{array}$ \\
\hline
\end{tabular}




\begin{tabular}{|l|l|}
\hline & $\begin{array}{l}\text { ofrecida a la biblioteca debe ser evaluada a fin de } \\
\text { conocer si los objetivos propuestos han sido logrados, } \\
\text { además de reflexionar sobre la experiencia. }\end{array}$ \\
\hline
\end{tabular}

Fuente: Elaboración propia.

\section{Camino a la gestión de la información}

La información dentro de las organizaciones se ha convertido en uno de los recursos prioritarios para la evolución y supervivencia de las mismas. Como tal, cumple con las siguientes particularidades: "que ésta sea administrada: es adquirida a un costo y tasable en dinero, tiene características específicas y mensurables, control del costo de la información, posee un ciclo de vida, capacidad de ser procesada y refinada, etc." (Bazán y otros, $s / f)$.

Mencionados en varios artículos, los objetivos de la gestión de recursos de información (GRI) hacen referencia a:

La maximización y beneficios derivados del uso de la información, minimizar el costo de adquisición, procesamiento y uso de la información, la posibilidad de determinar responsabilidades para el uso efectivo y eficiente de la información y asegurar un suministro continuo de información (Reyes Ramírez, 2007, p. 2).

Esto determina la relevancia de la información para ser usada en el proceso y perfeccionamiento de una organización para que alcance el éxito pretendido. En este sentido la gestión ha llegado a la información; Castillo Almeida y Pérez Rodríguez (2017), en su trabajo de investigación, afirman que esta misma gestión posibilita la toma de decisiones y facilita el desempeño del personal en la organización para cumplir con los objetivos.

Es real que el buen uso de la información optimiza las tareas, pero también es importante destacar que, en el equipo de trabajo, nace un cúmulo de información de sus relaciones personales vinculada a los conocimientos de cada uno de ellos y sus experiencias, y por ende no sólo interesa la información proveniente del exterior sino aquella que se genera de la misma interacción. Esta observación permite cruzar la frontera hacia la gestión del conocimiento, considerando al mismo como una amalgama de información, valores, conocimiento de contexto y experiencias que acumulan las personas en el desarrollo de sus tareas y que sirven para acometer nuevas experiencias (Alonso Arévalo, 2007, p. 7).

Middleton (1999) hace una distinción clara de la gestión de la información y el conocimiento, manifestando que éste último se utiliza habitualmente para describir los procedimientos para el uso óptimo del conocimiento del personal de la organización, y que el valor del conocimiento reside más en su uso que en su disponibilidad. En cambio, la información es un objeto en sí mismo y tiene una existencia independiente de su uso.

Por ende, la gestión del conocimiento tiene como propósito el diseño de estrategias, procesos, estructuras y sistemas que permiten a la organización hacer uso de lo que conoce, y la gestión de la información debe garantizar que la información esté disponible para cada persona en el momento requerido (Fernández Marcial, 2006).

El conocimiento, señala en sus estudios Bonilla (2003, p. 24), es una fuente de 
competitividad de gran valor para mejorar la calidad de los procesos, productos y servicios dentro de una organización, destinados éstos a dar respuesta a las demandas de los usuarios.

Rodríguez Cruz (2008) menciona que las organizaciones deben beneficiarse con la distribución y uso de la información, ya que el flujo de la misma permite una adecuación a los cambios que se abordan. A su vez, el autor pone énfasis en generar y consolidar una cultura informacional enfocada en generar ventajas.

Asimismo, y en razón de que las organizaciones se encuentran inmersas en la era de la información y el conocimiento, deben hacer frente a los nuevos escenarios de manera eficaz y eficiente, considerando además la inclusión de las tecnologías de la información como herramientas de apoyo (Vásquez, Rizo y Gabalán Coello, 2015).

La actividad desarrollada en la biblioteca popular, que comprendió uso de bibliografía pertinente, búsqueda de experiencias anteriores para conformar posteriormente las lecciones aprendidas, práctica de selección, análisis y evaluación de información, haciendo uso de capacidad crítica, reflexión, conocimientos y finalmente tomando decisiones, forma parte de esta gestión de la información y del conocimiento tan necesarias para las organizaciones. A continuación se detallan algunas de las actividades desarrolladas en las que se aplicaron herramientas para el uso y tratamiento de la información.

\section{Herramientas utilizadas para recuperar información}

\section{a) Análisis FODA}

Como actividad inicial, conocer la situación de la institución en la cual se desarrollará la práctica de extensión, resulta fundamental para direccionar luego las actividades mismas. Estar al tanto de la dinámica del medio indagando acerca de cómo está conformada la biblioteca desde el punto de vista de su personal, sobre cuál es su cultura organizacional, con qué recursos tanto materiales como financieros cuenta.

En definitiva, saber cuál es la dificultad con la que se encuentran en la actualidad.

Para ello se implementó una matriz FODA que reflejará claramente la situación sobre los libros y revistas donados y las consecuencias provocadas en la biblioteca, lo que permitirá identificar algunos problemas con los se encontrará el grupo y las posibilidades que se tendrán para concretar finalmente y con éxito la actividad. Los resultados se muestran en la Tabla 2. 
Tabla 2. FODA colecciones donadas a la Biblioteca Popular Atahualpa

\begin{tabular}{|c|c|c|}
\hline & Fortalezas & Debilidades \\
\hline $\begin{array}{l}\text { Factores } \\
\text { internos }\end{array}$ & $\begin{array}{l}\text { Donaciones } \\
\text { Personal predispuesto a } \\
\text { aprender } \\
\text { Libros con valor histórico. } \\
\text { Reconocimiento de la } \\
\text { necesidad de apoyo técnico. } \\
\text { Material de seguridad para } \\
\text { trabajar los estudiantes. } \\
\text { (guantes y barbijos) }\end{array}$ & $\begin{array}{l}\text { Espacio físico insuficiente } \\
\text { Personal no profesionalizado } \\
\text { Libros en mal estado } \\
\text { Apuntes donados } \\
\text { Revistas donadas } \\
\text { Metros lineales inutilizados } \\
\text { Cantidad de libros } \\
\text { No accesibilidad a los libros } \\
\text { donados. } \\
\text { Condiciones de limpieza de los } \\
\text { libros. } \\
\text { Ausencia de procedimientos } \\
\text { Ausencia de políticas }\end{array}$ \\
\hline & Oportunidades & Amenazas \\
\hline $\begin{array}{l}\text { Factores } \\
\text { externos }\end{array}$ & $\begin{array}{l}\text { Apoyo técnico de la Escuela } \\
\text { de Bibliotecología } \\
\text { Participación de estudiantes } \\
\text { formados en la temática. } \\
\text { Seguro de los participantes } \\
\text { otorgado por la } \\
\text { Universidad. }\end{array}$ & $\begin{array}{l}\text { Demora en la aprobación de la } \\
\text { propuesta. } \\
\text { Traslado de los participantes. } \\
\text { Pedido de los lectores por los } \\
\text { libros donados. } \\
\text { Diversidad de lectores }\end{array}$ \\
\hline
\end{tabular}

\section{b) Identificación de interesados y especificación de las necesidades}

A aquellas entidades consideradas como grupos de interés en un proyecto, se las denomina stakeholders. La Asociación Española de Contabilidad y Administración de Empresa (AECA) se ocupa de definirla, en su documento sobre el marco conceptual de la responsabilidad social corporativa (AECA, 2003), expresando que estos stakeholders comprenden aquellos grupos sociales o individuos afectados por la existencia y acción de una organización, que tienen un interés legítimo directo o indirecto e influyen en la consecución de sus objetivos (Liscano y Moneva, 2004).

De esta manera se decide como actividad inicial identificar a los interesados con los cuales se trabajará durante todo el recorrido del proyecto. Esta información obtenida indica que los grupos identificados son: los responsables del proyecto (docentes de la Escuela de Bibliotecología, de la FFyH-UNC), el equipo de trabajo (alumnos de la Escuela de Bibliotecología de la FFyH-UNC), dirigentes de la biblioteca popular, personal de la biblioteca, lectores e, indirectamente, la sociedad.

Generalmente, un proyecto comienza con una idea global, y para llevarlo a cabo es importante especificar sus requerimientos con el objeto de lograr minimizar los cambios posibles durante la ejecución. La información que contenga debe ser: necesaria, concisa, completa, consistente, verificable y sin ambigüedad. 
Entre las técnicas más usadas para el proceso de recolección de requerimientos, se pueden mencionar las entrevistas formales o informales, grupos de análisis o técnicas de creatividad grupal (como es la tormenta de ideas, la cual ha dado buenos resultados).

Estos requisitos expresan los deseos de los interesados, por ello la importancia de captar la real necesidad $y$, a partir de ello, planificar.

Una vez identificados, se procedió a analizar las expectativas de cada grupo. El tiempo y la profundidad de este análisis quedó en manos de los responsables del proyecto, dando por finalizado el mismo una vez obtenida la información necesaria para iniciar la acción de cambio.

En la tabla 3 se puede visualizar un resumen de las expectativas de cada uno de los grupos que dieron sentido a la dirección que luego tomó la propuesta.

Tabla 3. Registro de Expectativas de Grupos de Interés

\begin{tabular}{|c|c|}
\hline Grupos de interés & Expectativas \\
\hline $\begin{array}{l}\text { Responsables del } \\
\text { proyecto }\end{array}$ & $\begin{array}{l}\text { Cumplir con los objetivos propuestos en el } \\
\text { proyecto. } \\
\text { Contribuir solidariamente con entidades sin } \\
\text { fines de lucro. } \\
\text { Formar profesionales responsables y solidarios. } \\
\text { Involucrar a estudiantes de Bibliotecología en } \\
\text { la práctica temprana de la profesión. } \\
\text { Capacitar en prácticas específicas de la } \\
\text { bibliotecología }\end{array}$ \\
\hline Equipo de trabajo & $\begin{array}{l}\text { Aprender en la práctica lo estudiado en la } \\
\text { Escuela de Bibliotecología. } \\
\text { Contribuir con la sociedad por medio de } \\
\text { trabajo solidario. }\end{array}$ \\
\hline $\begin{array}{l}\text { Dirigentes de la } \\
\text { Biblioteca Popular }\end{array}$ & $\begin{array}{l}\text { Establecer vínculos con organismos educativos } \\
\text { que den respuesta a la diversidad de } \\
\text { necesidades de la biblioteca. } \\
\text { Concretar los propósitos planteados. } \\
\text { Mantener vías de comunicación permanente } \\
\text { con la Escuela de Bibliotecología. }\end{array}$ \\
\hline $\begin{array}{l}\text { Personal de la } \\
\text { Biblioteca Popular }\end{array}$ & $\begin{array}{l}\text { Formarse en prácticas bibliotecólogicas no } \\
\text { cotidianas. } \\
\text { Establecer vínculos con profesionales y } \\
\text { estudiantes en bibliotecología. }\end{array}$ \\
\hline Lectores & $\begin{array}{l}\text { Disponer de espacios cómodos y suficientes } \\
\text { para disfrutar de la lectura y actividades } \\
\text { culturales dentro de la biblioteca. }\end{array}$ \\
\hline Sociedad & $\begin{array}{l}\text { Aprovechar y disfrutar de manera adecuada los } \\
\text { servicios prestados por la biblioteca. }\end{array}$ \\
\hline
\end{tabular}

Fuente: Elaboración propia 
Como resultado de esta actividad, se pudo detectar por medio de estas expresiones el grado de compromiso de cada grupo. Este análisis también tiene utilidad para tomar decisiones en caso de aparición de variables motivacionales.

\section{c) Comunicación en el grupo}

Enfocados ya en la comunicación con los grupos de interés, se pensó un plan determinando acerca de: quién recibe la información, qué tipo de información, en qué momento, con qué periodicidad y a través de cuáles medios. Para ello se consideró que solamente se entregaría información precisa en función del rol que se cumplía.

Las modalidades de comunicación se limitaron a las siguientes:

- Informal escrita (mensajes por medio de telefonía móvil y correos electrónicos).

- Formal escrita (Presentación del proyecto en la Secretaría de Extensión de la FFyHUNC; Presentación de la propuesta en el Consejo de Escuela de Bibliotecología para su aprobación; Notas solicitando seguro de vida para los estudiantes participantes en el trabajo de campo; Nota de agradecimiento a la Biblioteca; Informe Técnico del trabajo realizado; Documento de Políticas de Análisis Crítico de la colección; Reporte a la Secretaria de Extensión por el trabajo y aporte realizado a la sociedad; Bibliografía para lectura de los estudiantes incorporada al aula virtual de la Facultad de Filosofía y Humanidades).

- Informal verbal (Primer encuentro con los dirigentes de la Biblioteca Popular; Reuniones periódicas de los responsables del proyecto).

- Formal verbal (Reunión con la Secretaría de Extensión de FFyH-UNC, planteando la necesidad de la biblioteca; Reunión con los docentes involucrados; Reunión con los alumnos involucrados; Reuniones con los dirigentes de la Biblioteca Popular).

El correo electrónico fue de mucha utilidad, sin embargo se evaluó a las reuniones como un espacio de mayor importancia, en tanto propicio para dialogar y discutir alguna dificultad surgida o decisión a tomar sobre la marcha.

En relación a la información proporcionada por el equipo responsable, para los alumnos se limitó a toda aquella vinculada al aspecto técnico para el desarrollo de las actividades, lo mismo para el personal de la biblioteca que participó; a los dirigentes de la biblioteca se comunicaban los informes de avances del trabajo y el resultado final; en relación a los usuarios y a la sociedad en general, la comunicación estuvo a cargo de la misma biblioteca, dando a conocer la incorporación de nuevo material bibliográfico que no podía ser exhibido debido a la reducción del espacio físico ocupado por las donaciones.

La comunicación en las reuniones fue fluida, permitiendo la participación en la toma de decisiones respecto a formas y modalidades en el trabajo y generando, de esta manera, un ámbito favorable en el grupo para avanzar adecuadamente y proyectar futuras actividades.

Vinculado a este tema, se destaca que la capacidad de comunicar de manera efectiva converge en la existencia de un clima propicio para el intercambio de ideas. Para ello, el 
responsable del proyecto debe asumir un rol fundamental como impulsor de creatividad que otorgue significado al trabajo del equipo.

En el caso particular de los alumnos participantes en este proyecto, se pensó en la realización de actividades en las cuales cada uno de ellos se sintiera cómodo y atraído a fin de que no surgieran conflictos como: frustración, desmotivación, falta de confianza, competitividad con sus pares, etc. Para ese fin se recabó información sobre preferencias y así poder distribuir las tareas.

Fue muy importante comunicar y ofrecer información acerca de la asignación de responsabilidades del grupo, para que cada uno claramente conociera su posición y cumpliera con la misma.

Se utilizó una matriz RACI en la cual queda reflejada la relación entre los paquetes de trabajo y los miembros del equipo. RACI es una sigla en inglés que significa:

R Responsible (responsable)

A Accountable (que rinde cuenta sobre la actividad)

C Consulted (a quien se puede consultar o se le solicita opinión)

I Informed (la persona que debe ser informada)

Se destaca, que, si alguna de las personas no está calificada para realizar cualquiera de las actividades asignadas, retrasará el proyecto. Por lo tanto es importante implementar dos modalidades de preguntas para evaluar el futuro desempeño de la persona; unas abiertas, que permitan obtener mayor información para su posterior análisis, y otras del tipo cerradas, más técnicas y específicas. La información que se obtenga de estas entrevistas será determinante para la distribución de las tareas y la formación de grupos de trabajo dentro del proyecto con intereses comunes.

Se señala que la utilidad de la competencia profesional está en la capacidad de ésta para hacer frente a los contextos profesionales cambiantes (Tejada Fernández y Navía Gómez, 2005). Estos mismos autores hacen mención de la competencia colectiva que involucra competencias relacionales y sociales, que van más allá de aquellas técnicas y procedimentales, y su complemento conduce a mejores resultados.

En la matriz RACI implementada se refleja el planteamiento anterior (Tabla 4): 
Tabla 4. Matriz RACI de Asignación de Responsabilidades

\begin{tabular}{|c|c|c|c|c|c|c|c|}
\hline Actividades & $\begin{array}{l}\text { Responsables } \\
\text { del proyecto }\end{array}$ & $\begin{array}{c}\text { Alumnos } \\
\text { participantes }\end{array}$ & $\begin{array}{l}\text { Personal } \\
\text { de la } \\
\text { Biblioteca }\end{array}$ & $\begin{array}{l}\text { Dirigentes } \\
\text { de la } \\
\text { Biblioteca }\end{array}$ & Lectores & $\begin{array}{l}\text { Secretaria } \\
\text { de } \\
\text { Extensión } \\
\text { de la } \\
\text { FFyH- } \\
\text { UNC }\end{array}$ & Sociedad \\
\hline $\begin{array}{c}\text { Reunión con } \\
\text { dirigentes de la } \\
\text { Biblioteca }\end{array}$ & $\mathrm{R}$ & & & c & & & \\
\hline $\begin{array}{l}\text { Reunión con la } \\
\text { Secretaria de } \\
\text { Extensión }\end{array}$ & $\mathrm{R}$ & & & & & I & \\
\hline $\begin{array}{l}\text { Reunión de } \\
\text { cátedras }\end{array}$ & $\mathrm{R}$ & 1 & & & & & \\
\hline $\begin{array}{l}\text { Diseño y } \\
\text { redacción del } \\
\text { proyecto }\end{array}$ & $\mathrm{R}$ & & & A & & A & \\
\hline $\begin{array}{l}\text { Comunicaciondel } \\
\text { proyecto }\end{array}$ & $\mathrm{R}$ & I & & I & & A & \\
\hline $\begin{array}{c}\text { Búsqueda de } \\
\text { bibliografía para } \\
\text { estudio }\end{array}$ & $R$ & $R$ & & I & & 1 & \\
\hline $\begin{array}{l}\text { Redaccion de } \\
\text { Políticas para la } \\
\text { bibliotecapopulary } \\
\text { formularios de } \\
\text { trabajo }\end{array}$ & $\mathrm{R}$ & $\mathrm{R}$ & C & c & & I & \\
\hline $\begin{array}{l}\text { Solicitud de } \\
\text { permiso para } \\
\text { traslado de } \\
\text { alumnos }\end{array}$ & $\mathrm{R}$ & I & 1 & 1 & & A & \\
\hline $\begin{array}{l}\text { Traslado de los } \\
\text { alumnos }\end{array}$ & $\mathrm{R}$ & I & I & 1 & & A & \\
\hline $\begin{array}{l}\text { Analisis critico de } \\
\text { la colección donada }\end{array}$ & $\mathrm{R}$ & $R$ & $\mathrm{R}$ & A & I & A & 1 \\
\hline $\begin{array}{l}\text { Controlde } \\
\text { cambios }\end{array}$ & $\mathrm{R}$ & & & I & & I & \\
\hline $\begin{array}{l}\text { Informe de } \\
\text { resultado de la } \\
\text { actividad }\end{array}$ & $\mathrm{R}$ & $R$ & I & I & I & I & I \\
\hline $\begin{array}{l}\text { Publicidad de la } \\
\text { actividades } \\
\text { desarrollada }\end{array}$ & 1 & 1 & 1 & $\mathrm{R}$ & I & 1 & 1 \\
\hline
\end{tabular}

Fuente: Elaboración propia.

Referencias: $\mathrm{R}=$ Ejecuta (Responsible, $\mathrm{A}=$ Supervisa (Accountable), $\mathrm{C}=$ Es consultado (Consulted), I = Es informado(Informed)

Además de la confección de esta matriz se especificaron los periodos de tiempo destinados para cada actividad, a fin de respetar el ciclo mismo del proyecto y el compromiso asumido.

\section{d) Flujograma}

Para un mejor seguimiento del proceso a desarrollar en la biblioteca popular, se decidió implementar un flujograma donde se refleja el conjunto de actividades estableciendo inicio y fin de cada una y, las relaciones entre los procesos, con la intención de mejorar prácticas de organización (Figura 1). 


\section{Figura 1. Flujograma Proyecto de Extensión}

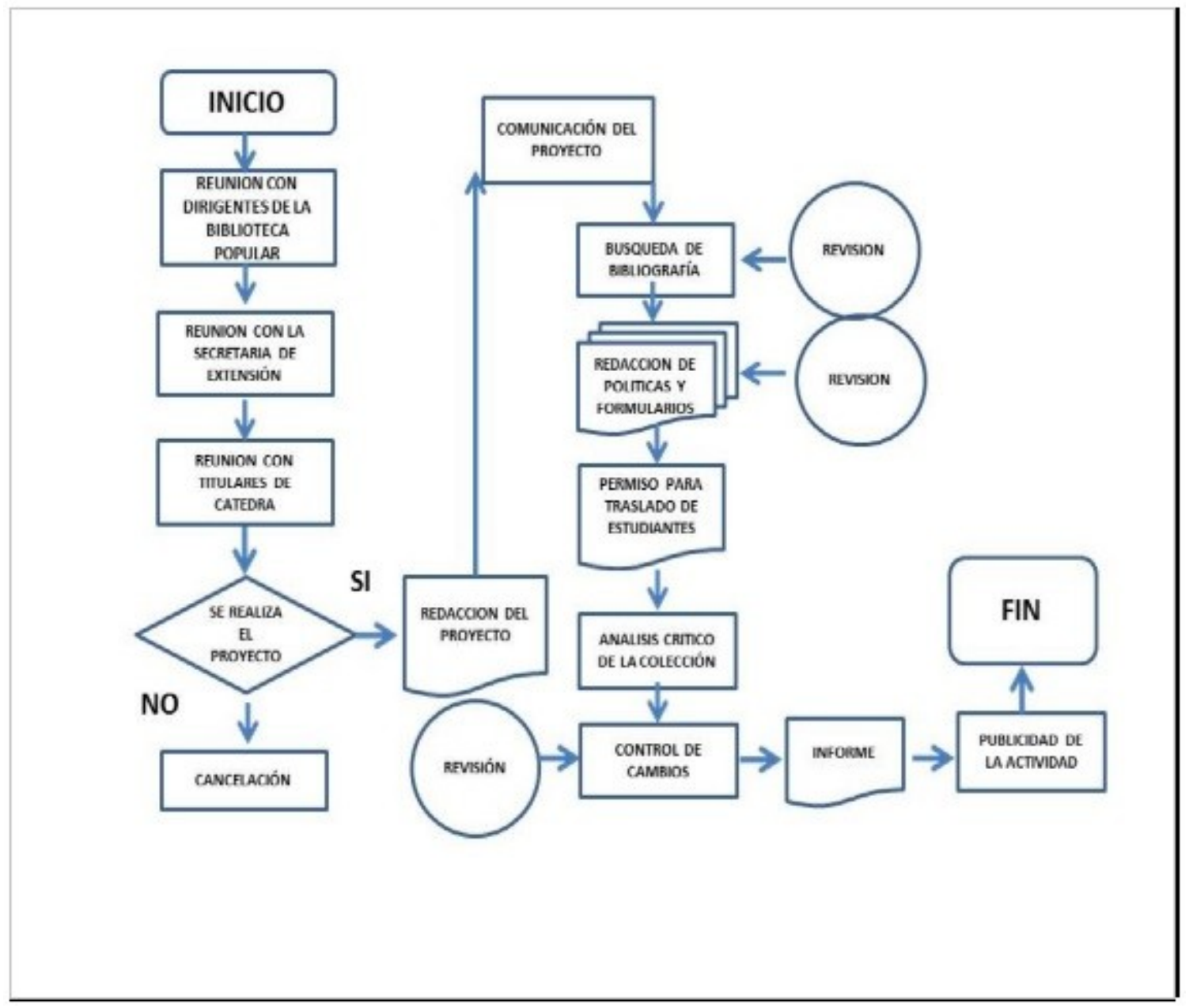

Fuente: Elaboración propia.

La información contenida en las distintas actividades que comprende el proceso cumple con un orden cronológico que los interesados deben conocer para desarrollar adecuadamente las actividades. Le agrega valor, ya que los procesos están disponibles para ser analizados, se pueden visualizar si las actividades están equilibradas o algunas de ellas resultan innecesarias. De esta manera se puede comprender el sistema de información de acuerdo a los procedimientos incorporados en el proceso.

\section{e) Lecciones aprendidas}

Otra herramienta utilizada para abordar la actividad ha sido la reconocida como lecciones aprendidas, desarrolladas en la planificación del proyecto. Para una mayor comprensión se acuerda en la siguiente definición:

Los documentos identificados como lecciones aprendidas contribuyen a convertir el conocimiento tácito (aquel que se encuentra en la mente y deriva de la experiencia de las personas), en conocimiento explícito (aquel contenido en documentos, archivos electrónicos u objetos) facilitando su difusión (Banco Interamericano de desarrollo ([BID], 2008). 
Específicamente, las lecciones aprendidas reflejan pautas generalizadas, producto de experiencias en la implementación de proyectos y donde la información contenida en ellas, es de vital importancia para la consecución de los mismos. Los logros y dificultades documentados son sustanciales en la generación de conocimientos, ya que producen un cambio en el modo de actuar. Si existe la posibilidad de identificar errores y conocer una buena práctica, la mejora y perfección en el ejercicio de la profesión será notoria en cada proyecto que se inicie.

¿Qué significado tiene esta práctica? Si se pone el foco en una buena práctica, identificada como una solución eficiente para una situación problemática puntual, es importante que aquellos que dirigen un proyecto decidan registrar lo experimentado en todo el ciclo del mismo. La oportunidad de poder consultar y reflexionar sobre otras experiencias, con la participación de los interesados, es un factor garantista en la gestión de esta información para poder tomar decisiones respecto a las vías de trabajo, sus procedimientos y la formación de aquellos que formarán parte del proyecto.

Estas lecciones pueden ser positivas o negativas; lo esencial es poder identificar en ellas cuáles han sido los factores de éxito y las deficiencias o riesgos para replicar o desechar procedimientos o decisiones según corresponda. En consecuencia, la información contenida en estas lecciones, reduce la incertidumbre y el tiempo utilizado para resolver factores críticos, sirve de apoyo para tomar una mejor decisión y por sobre todas las cosas genera conocimiento.

Hay diversas modalidades para identificar lecciones aprendidas. En el caso particular de este proyecto se optó por utilizar el observatorio de experiencias haciendo hincapié en prácticas anteriores similares, minimizando riesgos.

Para cumplir con procedimientos de gestión de información útil y dejar huella de lo realizado, el registro de experiencias de este proyecto en la biblioteca popular se realizó durante toda la trayectoria del mismo.

Se diseñó un formulario conteniendo información básica y aquella considerada relevante, respetando teniendo en cuenta los siguientes: los destinatarios, cuál es la lección aprendida, cuál es la contribución y el contexto de la lección.

La tabla 5 contiene la información básica del proyecto.

Tabla 5. Información básica del proyecto

\begin{tabular}{|l|l|}
\hline Contenido & Información \\
\hline $\begin{array}{l}\text { Nombre del } \\
\text { Proyecto }\end{array}$ & $\begin{array}{l}\text { Análisis Crítico de la colección en donaciones de la } \\
\text { Biblioteca Popular Atahualpa }\end{array}$ \\
\hline $\begin{array}{l}\text { Fecha de inicio y } \\
\text { cierre }\end{array}$ & Septiembre-Octubre del año 2016 \\
\hline $\begin{array}{l}\text { Responsable del } \\
\text { proyecto }\end{array}$ & $\begin{array}{l}\text { Docentes de las cátedras Administración de Recursos y } \\
\text { Servicios de Información II }\end{array}$ \\
\hline $\begin{array}{l}\text { Institución que } \\
\text { respalda el }\end{array}$ & $\begin{array}{l}\text { Escuela de Bibliotecología, Facultad de Filosofía y } \\
\text { Humanidades de la Universidad Nacional de Córdoba }\end{array}$ \\
\hline
\end{tabular}




\begin{tabular}{|l|l|}
\hline proyecto & \\
\hline Equipo de trabajo & Estudiantes y docentes de la Escuela de Bibliotecología \\
\hline Destinatarios & Biblioteca Popular Atahualpa \\
\hline
\end{tabular}

Fuente: Elaboración propia

En la tabla 6 se muestra uno de los registros de las actividades desarrolladas y cuyo formato ha sido utilizado para asentar las lecciones aprendidas.

Tabla 6. Información básica del proyecto

\begin{tabular}{|c|c|c|}
\hline Contenido & \multicolumn{2}{|c|}{ Información } \\
\hline $\mathrm{N}^{\circ}$ de Registro & \multicolumn{2}{|l|}{6} \\
\hline Tema & \multicolumn{2}{|l|}{ Bibliografía } \\
\hline Descripción de la actividad & \multicolumn{2}{|c|}{ Búsqueda y lectura de bibliografía pertinente } \\
\hline Acciones & \multicolumn{2}{|c|}{$\begin{array}{l}\text { Se limitó a la búsqueda de material bibliográfico } \\
\text { a fin de realizar lectura sobre contenidos } \\
\text { teóricos que fundamente la decisión en la } \\
\text { práctica. Se sugirió utilizar bibliografía que no } \\
\text { superara los } 5 \text { años de producción. }\end{array}$} \\
\hline Referencias & \multicolumn{2}{|c|}{ Mención del material leído } \\
\hline \multirow[t]{3}{*}{ Tipo de lección } & Experiencia exitosa & $\mathrm{X}$ \\
\hline & Riesgos & \\
\hline & Actividad recurrente & \\
\hline Resultados obtenidos & \multicolumn{2}{|c|}{$\begin{array}{l}\text { Se opta por diseñar una política respecto a la } \\
\text { recepción de donaciones para dar solución a la } \\
\text { Biblioteca Popular. Se establecen los criterios de } \\
\text { selección de expurgo del material donado y se } \\
\text { decidió elaborar un documento con información } \\
\text { de las actividades realizadas y sugerencias para } \\
\text { posteriores análisis críticos de colecciones. }\end{array}$} \\
\hline \multicolumn{3}{|l|}{ Firma del responsable } \\
\hline
\end{tabular}

Fuente: Elaboración propia

Tanto el uso de las lecciones aprendidas como el posterior registro de actividades, con las especificaciones propias de cada actividad, ha dado como resultado una nueva 
experiencia, beneficiosa en todo su empleo.

\section{Conclusión}

Es evidente que la gestión no sólo es aplicable a los recursos humanos, materiales y financieros, ya que la información se ha venido posicionando de manera tal que sin ella ya no pueden tomarse decisiones.

En los proyectos se debe optar por distintas alternativas que hacen necesaria la información para intervenir. Es en esa actuación donde se crea un proceso cíclico necesario, en que una información va sumándose a otras.

Entre las herramientas implementadas en este proyecto de extensión, se tiene el caso de la matriz FODA en primera instancia, que ha permitido reconocer aquellas debilidades existentes a través de la información contenida en dicha matriz, pudiendo diferenciar situaciones problemáticas urgentes de las prioritarias y de esta manera enfocar el desarrollo de las actividades sobre aspectos edilicios en relación al insuficiente espacio físico, y sobre el análisis y destino de las colecciones donadas para dar respuesta a la biblioteca.

En segundo lugar, la RACI ha sido un instrumento necesario para definir el grado de responsabilidad de cada uno de los participantes en los procesos o actividades; permitió además conocer de manera rápida y eficaz a los mismos, localizando la información con facilidad.

El flujograma como guía del proceso de las actividades, facilitó la gestión; esto debido a que la visualización del mismo e interpretación generó acuerdos entre los participantes en cuanto a las metodologías a implementar, ya sea para la utilización de recursos, los tiempos empleados y también para la coordinación de las tareas.

En relación al uso de las lecciones aprendidas, práctica utilizada por primera vez, ha sido un importante aprendizaje, pudiendo aplicar experiencias anteriores y haciendo visible las acciones y resultados obtenidos en este proyecto particular. Lo fundamental de transmitir y mantener un canal de comunicación concreto ha encontrado, en este instrumento, un espacio de participación e intercambio de experiencias entre alumnos, docentes y demás involucrados.

La razón de este trabajo reside en el buen uso y tratamiento que se realice de la información. En el proyecto de extensión descripto en este artículo, la información utilizada ha traído como resultado una adecuada aplicación de los procedimientos desarrollados, un acercamiento casi total a decisiones acertadas para llevar adelante el proceso del proyecto. No obstante ello, algunas cuestiones referentes a la organización de las actividades en los tiempos establecidos, debieron mejorarse.

Otro punto a destacar es la importancia de la calidad y veracidad de la información, no sólo en cuanto a actualización sino a contenido, ya que ello incrementa los conocimientos en el ámbito de desempeño. En razón de ello, las fuentes deben cumplir con la particularidad de ser fiables como garantía a lo realizado o decidido.

El empoderamiento y protagonismo que ha adquirido en los últimos tiempos la 
información impulsada por la tecnología es indiscutible. Pero, de a poco los individuos dentro del ámbito de las instituciones van incorporando habilidades vinculadas a la interpretación de aquello que es conveniente y veraz, dejando en otro costal lo anecdótico.

Por otro lado, gestionar el cúmulo de información en la actualidad no es tarea fácil, y por ello se debe poner el esfuerzo en implementar estrategias comunicativas que den como resultado una adecuada información aprovechando las oportunidades que brindan las tecnologías.

Finalmente, existe un proceso de transformación de la cultura por un consumo desmedido de información, que en su mayor parte no resulta beneficioso. Por ello, se apunta al desarrollo de capacidades destinadas al análisis y evaluación de este recurso tan discutido y valorado en la actualidad.

Como resultado de esta práctica de extensión en la biblioteca intervenida, se realizó el análisis crítico de las colecciones redactando un plan de expurgo donde se establecían criterios de selección y destino del material. Se recuperaron metros lineales para poner a disposición libros nuevos y usados, de acuerdo a lo solicitado inicialmente por la Biblioteca Popular Atahualpa.

\section{Referencias bibliográficas}

AECA. Comisión de Responsabilidad Social Corporativa. (2003). Marco conceptual de la responsabilidad social y corporativa. Documento AECA (1). Recuperado de: www3.uji.es/ munoz/AECA.DOC

Alonso Arévalo, J. (2007). Gestión de la información, gestión de contenidos y conocimiento. En II Jornadas de Trabajo del Grupo SIOU. Salamanca, España. Recuperado de: http://eprints.rclis.org/11273/

Bazán, I. et.al. (s/f). La gestión de los recursos de información: Importancia, desafíos y responsabilidades. Recuperado de:

http://www.mdp.edu.ar/humanidades/documentacion/licad/archivos/modulos/inicial/archi vos/bibliografia/inicial/eje3/ADMINISTRACION/UNIDADES DE INFORMACION/MI080.htm

BID. (2008). Notas de lecciones aprendidas. Recuperado de: http://boru.pbworks.com/f/Lecciones+Aprendidas+11-18-08.pdf

Bonilla, G. (2003). Sociedad de la información y gestores de la información. Biblios, 4(16). Recuperado de: https://dialnet.unirioja.es/servlet/articulo?codigo $=759378$

Castillo Almeida, G. y Pérez Rodríguez, E. (2017). Diagnóstico de los sistemas de información en las empresas priorizadas según los requerimientos actuales. Palabra clave, 6(2), e022. Recuperado de: http://www.palabraclave.fahce.unlp.edu.ar/article/view/PCe022/8122 
Fernández Marcial, V. (2006). Gestión del conocimiento versus gestión de la información. Investigación bibliotecológica, 20(41). Recuperado de: http://www.scielo.org.mx/scielo.php?script=sci arttext\&pid=S0187-358X2006000200003 Liscano, J. y Moneva, J. (2004). Marco conceptual de la responsabilidad corporativa. En XI Encuentro AECA. NIF y otros retos de la gestión empresarial. Madrid: Asociación Española de Contabilidad y Administración de Empresas. Recuperado de: http://www.aeca1.org/revistaeca/revista68/68.pdf

Middleton, M. (1999). De la gestión de la información a la gestión del conocimiento: perspectivas sobre el desarrollo. El profesional de la información, 8(5). Recuperado de: http://www.elprofesionaldelainformacion.com/contenidos/1999/mayo/de la gestin de la informacin_a_la_gestin_del_conocimiento_perspectivas_sobre_el desarrollo.html

Reyes Ramírez, L. (2007). Sistemas de información para la prensa: la gestión de la información y el conocimiento en el contexto de los sistemas integrados de información. ACIMED, 15(2). Recuperado de: http://scielo.sld.cu/scielo.php? script=sci arttext\&pid=S1024-94352007000200004

Rodríguez Cruz, Y. (2008). Gestión de información e inteligencia: integración en los contextos organizacionales. ACIMED, 17(5). Recuperado de http://scielo.sld.cu/scielo.php? script $=$ sci arttext $\&$ pid $=$ S1024-94352008000500003

Tejada Fernández, J. y Navía Gámez, A. (2005). El desarrollo y la gestión de competencias profesionales: una mirada desde la formación. Revista iberoamericana de educación, 37(2). Recuperado de http://rieoei.org/1089.htm

Vásquez Rizo, F. E. y Gabalán Coello, J. (2015). Información y ventaja competitiva, coexistencia exitosa en las organizaciones de vanguardia. El profesional de la información, 24(2). Recuperado de http://www.elprofesionaldelainformacion.com/contenidos/2015/mar/08.html 